\title{
LINEAR DIFFERENTIAL EQUATIONS WHERE NONOSCILLATION IS EQUIVALENT TO EVENTUAL DISCONJUGACY
}

\author{
JERRY R. RIDENHOUR
}

\begin{abstract}
Conditions on $n$th order linear differential equations are given which imply that nonoscillation is equivalent to eventual disconjugacy. These conditions are in the form of assumptions that certain boundary-value functions are infinite for all values of the argument.
\end{abstract}

1. Introduction. We are concerned with the real $n$th order linear differential equation

$$
y^{(n)}+p_{n-1}(x) y^{(n-1)}+\cdots+p_{0}(x) y=0
$$

on the interval $(a, \infty)$ where $n \geq 3,-\infty \leq a<\infty$, and $p_{i} \in C(a, \infty), i=0, \cdots$, $n-1$. Equation (1) is said to be oscillatory if there is a nontrivial (not identically zero) solution of (1) whose set of zeros is unbounded above, and is said to be nonoscillatory otherwise. Equation (1) is said to be conjugate on an interval $I$ if there is a nontrivial solution of (1) which has at least $n$ zeros counting multiplicities on $I$, and is said to be disconjugate on $I$ if this is not the case. Further, equation (1) is said to be eventually disconjugate if there exists a number $t$ in $(a, \infty)$ such that $(1)$ is disconjugate on $[t, \infty)$. The known properties of second order equations, the paper on fourth order equations by Leighton and Nehari [6], and the work on third order equations by Hanan [3] all pointed to the possibility that nonoscillation is equivalent to eventual disconjugacy for general $n$th order equations; however, this question was settled in the negative with counterexamples given by Gustafson [1], [2]. The main result of this paper gives sufficient conditions under which nonoscillation is equivalent to eventual disconjugacy. More explicitly, we will show that nonoscillation is equivalent to eventual disconjugacy when equation (1) satisfies

Received by the editors October 10, 1973 and, in revised form, March 20, 1974. AMS (MOS) subject classifications (1970). Primary 34C10; Secondary 34B05, 34B10, 34A30.

Key words and phrases. Linear differential equations, oscillation, nonoscillation, conjugacy, disconjugacy, eventual disconjugacy, $k$ th conjugate point, extremal solutions, boundary-value functions, distributions of zeros. 


$$
s_{p}^{2}=r(p, n-p+1)=r(p+1, n-p)=r(p, 2, n-p)=\infty
$$

for some $p$ with $1 \leq p \leq n-1$ (appropriate definitions are given in $\$ 2$ ).

These results can be viewed as $n$th order extensions of certain fourth order results of Leighton and Nehari [6] and the third order results of Hanan [3]. Furthermore, this completes a theory through which it seems like ly that sufficient conditions for oscillation of higher order equations can be obtained (this is elaborated on in comments following the corollary to Theorem 3 ).

2. Definitions and agreements. By a solution of (1), we will always mean a nontrivial solution of (1). If $y(x)$ is a solution of (1) and $\alpha \in(a, \infty)$, we will use the notation $[a ; y]$ to denote the exact multiplicity of $y(x)$ at $x=$ $\alpha$; that is, $[\alpha ; y]$ is the smallest nonnegative integer $m$ such that $y^{(m)}(\alpha)$ $\neq 0$ (here $y^{(0)}(\alpha)=y(\alpha)$ ). Any reference to the number of zeros of a solution of (1) will be a reference to the number of zeros counting multiplicities unless it is stated that distinct zeros are being considered.

If $i_{1}, \cdots, i_{k}$ are positive integers with $i_{1}+\cdots+i_{k} \geq n$, then a solution $y(x)$ of $(1)$ is said to have an $\left(i_{1}, \cdots, i_{k}\right)$-distribution of zeros (or, more briefly, $\left(i_{1}, \cdots, i_{k}\right)$-zeros $)$ on an interval $I$ if there exist $k$ points $a_{1}, \ldots$, $a_{k}$ in $I$ with $\alpha_{1}<\cdots<\alpha_{k}$ and $\left[\alpha_{j} ; y\right] \geq i_{j}(j=1, \cdots, k)$. Suppose now that $t \in(\alpha, \infty)$. As a matter of convention, take the infimum of the empty set to be $\infty$ in the following definitions. If $i_{1}, \cdots, i_{k}$ are positive integers with $i_{1}+\cdots+i_{k} \geq n$, let $r\left(i_{1}, \cdots, i_{k} ; t\right)$ be the infimum of all numbers $c>$ $t$ such that (1) has a solution with $\left(i_{1}, \ldots, i_{k}\right)$-zeros on the interval $[t, c]$. If $p$ is an integer with $1 \leq p \leq n-1$, take $s_{p}^{2}(t)$ to be the infimum of all numbers $c>t$ such that (1) has a solution with $\left(i_{1}, \cdots, i_{k}\right)$-zeros on $[t, c]$ where $i_{1}, \cdots, i_{k}$ satisfy $k=n-1, i_{j}=1$ for $j \neq p$, and $i_{p}=2$. If $p$ and $q$ are positive integers with $q \leq n-1$ and $p+q \leq n+1$, let $N_{p}^{q}(t)$ be the infimum of all numbers $c>t$ such that $(1)$ has a solution with $\left(i_{1}, \ldots, i_{k}\right)$-zeros on $[t, c]$ where $i_{1}, \cdots, i_{k}$ satisfy $\Sigma_{j=1}^{s-1} i_{j} \geq p-1, i_{s} \geq q$, and $\sum_{j=s+1}^{k} i_{j} \geq n-$ $p-q+1$ for some integer $s$ with $1 \leq s \leq k$ (here we take $\sum_{j=1}^{0} i_{j}=\sum_{j=k+1}^{k} i_{j}=$ 0 when $s=1$ or $s=k$ ). Note that $s_{p}^{2}(t)$ depends on zero-distributions where the $p$ th distinct zero is of multiplicity at least two and there are at least $p-1$ distinct zeros to the left of and at least $n-p-1$ distinct zeros to the right of the $p$ th distinct zero, while $N_{p}^{q}(t)$ depends on zero-distributions where the pth zero is of multiplicity at least $q$ and there are at least $p-1$ zeros counting multiplicities to the left of and at least $n-p-q+1$ zeros counting multiplicities to the right of the $p$ th zero; hence, the notations $s_{p}^{2}(t)$ and $N_{p}^{q}(t)$ are descriptive of their respective meanings. By $r\left(i_{1}, \cdots, i_{k}\right)=\infty$, 
we mean that $r\left(i_{1}, \ldots, i_{k} ; t\right)=\infty$ for all $t \in(a, \infty)$; likewise, $s_{p}^{2}=\infty$ and $N_{p}^{q}=\infty$ mean that $s_{p}^{2}(t)=\infty$ for all $t \in(a, \infty)$ and $N_{p}^{q}(t)=\infty$ for all $t \in(a, \infty)$, respectively.

The boundary-value functions $r\left(i_{1}, \ldots, i_{k} ; \cdot\right), s_{p}^{2}$, and $N_{p}^{q}$ are studied in [8]. Although the list is not complete, several references to previous work on $r\left(i_{1}, \ldots, i_{k} ; \cdot\right)$ and $s_{p}^{2}$ are also in [8].

If $t \in(a, \infty)$ and $k$ is a positive integer, define the kth conjugate point of $t$, denoted by $\eta_{k}(t)$, to be the infimum of all numbers $c>t$ such that there is a solution of (1) with a zero at $t$ and at least $n+k-1$ zeros on $[t, c]$ (take $\eta_{k}(t)=\infty$ if no such $c$ exists). It is well known that this infimum is actually a minimum when $\eta_{k}(t)<\infty$; that is, solutions do exist having a zero at $t$ and at least $n+k-1$ zeros on $\left[t, \eta_{k}(t)\right]$ when $\eta_{k}(t)<\infty$. All such solutions are called extremal solutions for $\eta_{k}(t)$. The conjugate point functions have been studied by many authors, and the reader is referred to [8] for a partial list of references.

3. Main results. The results to be proved here depend on theory developed by the author in [8] and on the recent work of M. S. Keener [5]. Also, a basic tool is the following "double-zero lemma" which has been used by many authors (for a proof, see [9]).

Lemma 1. Suppose $a<\alpha<\beta<\infty$ and $u(x)$ and $v(x)$ are solutions of (1) with $[\alpha ; u]>[\alpha ; v]$ and $[\beta ; u]>[\beta ; v]$. If $v(x) \neq 0$ for $x \in(\alpha, \beta)$, then there is a constant $k$ and a number $\lambda$ in $(\alpha, \beta)$ such that $[\lambda ; u+k v] \geq 2$.

The following three lemmas are specializations of results in [8]. More specific reference to $[8]$ is made after these results are stated.

Lemma 2. If equation (1) satisfies $(*)$ for some $p$ and $i$ and $k$ satisfy $i+k \geq p+2$ and $i \leq p$, then $N_{i}^{k}=\infty$.

Lemma 3. If equation (1) satisfies (*) for some $p, \eta_{k}(t)<\infty$, and $y(x)$ is an extremal solution for $\eta_{k}(t)$, then $[t ; y]=p,\left[\eta_{k}(t) ; y\right]=n-p$, and $y(x)$ has exactly $k-1$ distinct zeros in $\left(t, \eta_{k}(t)\right)$, each of which is a simple zero.

Lemma 4. If equation (1) satisfies (*) for some $p$ and $k$ is a given positive integer, then $\left\{t \mid \eta_{k}(t)<\infty\right\}$ is the interval $(a, L)$ for some $L$ with $a \leq L \leq \infty$, and $\eta_{k}$ is a strictly increasing continuous function on $(a, L)$.

Lemma 2 follows from Theorem 2.3 of [8] in case $k \geq 3$ and from Lemma 4.1 of [8] in case $k=2$ and $2 \leq p \leq n-2$; however, no result in [8] immediately implies Lemma 2 when $k=2$ and $p=1$ or $p=n-1$. Lemma 
2 is trivial when $n=3$. If $n>3, p=1$, and $k=2$, then Theorem 2.3 of [8] implies $N_{1}^{3}=\infty$, conclusion (1) of Theorem 2.2 of [8] implies $r(2, n-2)=\infty$, and Lemma 2.4 of [8] implies $N_{1}^{2}=\infty$. Similarly, Theorem 2.3 of [8], conclusion (3) of Theorem 2.2 of [8], and Lemma 2.4 of [8] imply $N_{n-1}^{2}=\infty$ when $n>3, p=n-1$, and $k=2$; hence, Lemma 2 is true in any case. Lemma 3 follows from Theorem 4.1 of [8] when $p=1$ or $p=n-1$ and from Theorem 4.2 of [8] and the remark following Theorem 4.3 of [8] when $2 \leq$ $p \leq n-2$. Lemma 4 follows from Theorem 4.1 of [8] when $p=1$ or $p=n$ 1 , and from Theorem 4.3 of [8] and the remark following Theorem 4.3 of [8] when $2 \leq p \leq n-2$.

The following theorem is basic to the rest of the paper and appears in the recent work of M. S. Keener [5]. It is an easy consequence of Lemma 2, and a proof is given here for the sake of completeness.

Theorem 1. If equation (1) satisfies $(*)$ for some $p$ and $y(x)$ is a solution of (1) with $\left(i_{1}, \ldots, i_{k}\right)$-zeros on $(a, \infty)$, then there exist integers $q$ and $r$ with $1 \leq q<r \leq k, i_{1}+\cdots+i_{q}=p$, and $i_{r}+\cdots+i_{k}=n-p$.

Proof. Let $a_{1}, \cdots, a_{k}$ be such that $a<\alpha_{1}<\cdots<a_{k}<\infty$ and $\left[\alpha_{j} ; y\right] \geq i_{j}(j=1, \cdots, k)$. Let $q=\min \left\{j \mid i_{1}+\cdots+i_{j} \geq p\right\}$. Suppose that $i_{1}+\cdots+i_{q}>p$. Let $K=i_{1}+\cdots+i_{q}$. If $q=1$, then $y(x)$ has $N_{1}^{K}$-zeros on $(a, \infty)$; however, $1 \leq p$ and $K+1 \geq p+2$, thus contradicting Lemma 2 . Hence, $q>1$ so let $L=i_{1}+\cdots+i_{q-1}$. Now $L \leq p-1$ and $y(x)$ has $N_{L+1}^{i} q$-zeros on $(a, \infty)$, again contradicting Lemma 2 since $L+1 \leq p$ and $i_{q}+L+1 \geq p+2$. This shows that $i_{1}+\cdots+i_{q}=p$.

A similar proof shows that $i_{r}+\cdots+i_{k}=n-p$, and it then follows that $1 \leq q<r \leq k$.

Let $\eta_{1}^{k}, k=1,2,3, \cdots$, be the composition functions defined by $\eta_{1}^{1}=$ $\eta_{1}$ and $\eta_{1}^{i}=\eta_{1} \circ \eta_{1}^{i-1}$ for $i>1$. The following theorem is of interest in itself.

Theorem 2. If equation (1) satisfies ( $*$ ) for some $p$ and $t \in(a, \infty)$, then $\eta_{k}(t) \leq \eta_{1}^{k}(t)(k=1,2, \ldots)$.

Proof. Suppose $t \in(a, \infty)$. The proof is by induction. The conclusion is trivially true when $k=1$, so assume $k>1$ and $\eta_{k-1}(t) \leq \eta_{1}^{k-1}(t)$. We wish to prove that $\eta_{k}(t) \leq \eta_{1}^{k}(t)$. Assume $\eta_{1}^{k}(t)<\infty$, since there is nothing to prove otherwise. By Lemma $4, \eta_{k-1}$ is a strictly increasing continuous function since equation (1) satisfies (*). Hence, $\eta_{k-1}(t) \leq \eta_{1}^{k-1}(t)<\eta_{1}^{k}(t)$ $<\infty$ implies there is a point $\alpha$ in the interval $\left[t, \eta_{1}^{k-1}(t)\right)$ such that $\eta_{k-1}(\alpha)$ $=\eta_{1}^{k-1}(t)$.

For notational purposes, let $\beta=\eta_{1}^{k-1}(t)=\eta_{k-1}(\alpha)$ and let $\gamma=\eta_{1}^{k}(t)=$ 
$\eta_{1}(\beta)$. Since $\eta_{k}$ is increasing, we need only to show that there is a solution of (1) having at least $n+k-1$ zeros on $[t, \gamma]$.

Let $u(x)$ be an extremal solution for $\eta_{k-1}(\alpha)$ and let $v(x)$ be an extremal solution for $\eta_{1}(\beta)$. By Lemma $3,[\alpha ; u]=[\beta ; v]=p,[\beta ; u]=[\gamma ; v]=n-p$, $u(x)$ has exactly $k-2$ distinct zeros in $(\alpha, \beta)$ each of which is a simple zero, and $v(x) \neq 0$ for $x$ in $(\beta, \gamma)$.

Suppose for now that $p$ satisfies $1<p<n-1$. The cases where $p=1$ or $p=n-1$ will be discussed later. Let $u_{1}(x), \ldots, u_{n-p-1}(x)$ be solutions of $(1)$ such that $u_{j}(x)$ for $1 \leq j \leq n-p-1$ satisfies $\left[\alpha ; u_{j}\right] \geq p-1,\left[\beta ; u_{j}\right]$ $\geq n-p-j$, and $\left[\gamma ; u_{j}\right] \geq j$. Theorem 1 implies that $\left[\beta ; u_{j}\right]=n-p-j$ for $j=1, \cdots, n-p-1$. Also, $\left[\alpha ; u_{1}\right]=p-1$, for otherwise $u^{(p)}(\alpha) u_{1}(x)-$ $u_{1}^{(p)}(\alpha) u(x)$ contradicts Theorem 1 . If $k=2$, then Lemma 1 and Theorem 1 together imply that $u_{1}(x)$ must have at least one zero in $(\alpha, \beta)$. If $k \geq 3$, let $\alpha_{1}, \ldots, \alpha_{k-2}$ where $\alpha<\alpha_{1}<\cdots<a_{k-2}<\beta$ be all the distinct zeros of $u(x)$ in $(\alpha, \beta)$, each of which we know to be a simple zero. Theorem 1 implies that $u_{1}\left(\alpha_{i}\right) \neq 0(i=1, \cdots, k-2)$. By Lemma $1, u_{1}(x)$ must have a zero in each of the intervals $\left(\alpha, \alpha_{1}\right)$ and $\left(\alpha_{k-2}, \beta\right)$. Also, when $k \geq 4$, $u_{1}(x)$ must have a zero in each of the intervals $\left(\alpha_{j}, \alpha_{j+1}\right), j=1, \ldots, k-3$. Hence, regardless of the value of $k$, there exist $k-1$ points $\alpha_{11}, \ldots$, $a_{1(k-1)}$ such that $a<\alpha_{11}<\cdots<a_{1(k-1)}<\beta$ and $u_{1}\left(a_{1 j}\right)=0(j=1, \cdots$, $k-1)$.

Using induction and comparing the zeros of $u_{i}(x)$ with those of $u_{i-1}(x)$ when $i>1$, we see that, for each $i$ with $1 \leq i \leq n-p-1$, there exist $k-1$ points $\alpha_{i 1}, \cdots, \alpha_{i(k-1)}$ such that $\alpha<\alpha_{i 1}<\cdots<\alpha_{i(k-1)}<\beta$ and $u_{i}\left(\alpha_{i j}\right)$ $=0, j=1, \cdots, k-1$.

For each $j$ with $1 \leq j \leq p-1$, let $v_{j}(x)$ be a solution of (1) such that $\left[\alpha ; v_{j}\right] \geq j,\left[\beta ; v_{j}\right] \geq p-j$, and $\left[\gamma ; v_{j}\right] \geq n-p-1$. Using Theorem 1 , we see that $\left[\beta ; v_{j}\right]=p-j(j=1, \cdots, p-1)$ and that $\left[\gamma ; v_{1}\right]=n-p-1$. It follows from Lemma 1 , Theorem 1 , and proof by induction that there exist $p-1$ points $\beta_{1}, \cdots, \beta_{p-1}$ in $(\beta, \gamma)$ such that $v_{j}\left(\beta_{j}\right)=0, j=1, \cdots, p-1$.

The solutions $u_{n-p-1}(x)$ and $v_{p-1}(x)$ are such that $\left[\alpha ; u_{n-p-1}\right] \geq$ $p-1,\left[\alpha_{(n-p-1) j} ; u_{n-p-1}\right] \geq 1(j=1, \cdots, k-1), \alpha<\alpha_{(n-p-1) 1}<\cdots<$ $a_{(n-p-1)(k-1)}<\beta,\left[\beta ; u_{n-p-1}\right]=1,\left[\gamma ; u_{n-p-1}\right] \geq n-p-1,\left[\alpha ; v_{p-1}\right] \geq p-1$, $\left[\beta ; v_{p-1}\right]=1,\left[\beta_{p-1} ; v_{p-1}\right] \geq 1, \beta<\beta_{p-1}<\gamma$, and $\left[\gamma ; v_{p-1}\right] \geq n-p-1$. If the solutions $u_{n-p-1}(x)$ and $v_{p-1}(x)$ were linearly independent, then

$$
u_{n-p-1}^{\prime}(\beta) v_{p-1}(x)-v_{p-1}^{\prime}(\beta) u_{n-p-1}(x)
$$

would be a solution of (1) having a $(p-1,2, n-p-1)$-distribution of zeros, thus contradicting Theorem 1 ; hence, $u_{n-p-1}(x)$ must be a constant multiple 
of $v_{p-1}(x)$. We see that $u_{n-p-1}(x)$ has at least $n+k-1$ zeros on $[a, \gamma]$, which shows that $n_{k}(t) \leq y$ when $1<p<n-1$.

In the case where $p=1$, let the solutions $u_{1}(x), \cdots, u_{n-p-1}(x)$ be defined as when $1<p<n-1$. We see as before that $u_{n-p-1}(x)$ has $\left[\beta ; u_{n-p-1}\right]=1$ and at least $k-1$ distinct zeros in $(\alpha, \beta)$. Theorem 1 then implies that $u_{n-p-1}(x)$ and $v(x)$ must be linearly dependent; hence, $v(x)$ has at least $n+k-1$ zeros on the interval $(\alpha, \gamma]$. When $p=n-1$, the solutions $v_{1}(x), \cdots, v_{p-1}(x)$ are defined as when $1<p<n-1$. It follows that $\left[\beta ; v_{p-1}\right]=1, v_{p-1}(x)$ has at least one zero in $(\beta, \gamma)$, and $v_{p-1}(x)$ and $u(x)$ are linearly dependent; hence, $u(x)$ has at least $n+k-1$ zeros on the interval $[\alpha, \gamma)$.

In any case, there exists at least one solution of (1) having at least $n+k-1$ zeros on the interval $[\alpha, \gamma]$. This completes the proof.

Remark. Since the conjugate point functions are increasing continuous functions when equation (1) satisfies (*), we see by inspecting the above proof that the conclusion of The orem 2 can be improved to $\eta_{k}(t)<\eta_{1}^{k}(t)$ when $k>1, \eta_{1}^{k}(t)<\infty$, and $p=1$ or $p=n-1$.

Theorem 3, the main result, will follow from Theorem 2 and a recent result of M. S. Keener [5] which we state here as Lemma 5.

Lemma 5 (M. S. Keener [5]). If equation (1) satisfies ( $*$ ) for some $p$, then (1) is oscillatory if there is a point $b$ in $(a, \infty)$ such that $\eta_{k}(b)<\infty$, $k=1,2, \cdots$.

Theorem 3. If equation (1) satisfies (*) for some $p$, then (1) is oscillatory if and only if $\eta_{1}(t)<\infty$ for all $t \in(a, \infty)$.

Proof. If there is an oscillatory solution of (1), then, given $t \in(a, \infty)$, there is a solution of (1) with at least $n$ zeros on $(t, \infty)$. Since $\eta_{1}$ is known to be an increasing function, it follows that $\eta_{1}(t)<\infty$.

On the other hand, if $\eta_{1}(t)<\infty$ for all $t \in(a, \infty)$, then $\eta_{k}(t) \leq \eta_{1}^{k}(t)<\infty$ for all $t \in(a, \infty), k=1,2, \cdots$. Hence, (1) is oscillatory by Lemma 5 .

As already mentioned, Theorem 3 implies that nonoscillation is equivalent to eventual disconjugacy when equation (1) satisfies (*). Furthermore, since $\eta_{1}(t)=r(p, n-p ; t)$ when equation (1) satisfies $(*)$, we state an immediate corollary of Theorem 3 .

Corollary. If equation (1) satisfies (*) for some $p$, then (1) is oscillatory if and only if $r(p, n-p ; t)<\infty$ for all $t \in(a, \infty)$.

It seems likely that this corollary may lead to direct conditions on the coefficients which are sufficient for (1) to be oscillatory; for if conditions 
are found which guarantee that equation (1) satisfies $(*)$, then the problem of proving (1) is oscillatory reduces to one of showing $r(p, n-p ; t)<\infty$ for all $t \in(a, \infty)$. Nehari [7] has proved a result which indicates that there is a large class of equations for which solutions cannot have more than $n$ zeros at two points; hence, the condition $r(p, n-p+1)=r(p+1, n-p)=\infty$ does not seem too restrictive. Furthermore, if $s_{p}^{2}=\infty$, Lemma 4.1 of [8] indicates that it is reasonable to suspect that $r(p, 2, n-p)=\infty$.

The case where $p=n-1$ seems particularly promising since $(*)$ reduces to $s_{n-1}^{2}=r(n-1,2)=\infty$; furthermore, $r(p, n-p ; t)=r(n-1,1 ; t)$ so $(1)$ is oscillatory if the solution $y(x)$ satisfying the initial conditions $y^{(i)}(t)=$ $\delta_{i(n-1)}$ has at least one zero in $(t, \infty)$.

It should be noted that selfadjoint equations with $s_{p}^{2}=\infty$ and $p \neq n / 2$ are necessarily disconjugate on $(a, \infty)$ by Corollary 3.2.1 of [8]. Oscillation conditions could be found for selfadjoint equations by the above procedure only when $p=n / 2$; however, the already mentioned results of Leighton and Nehari [6] do provide examples of this type.

Finally, we remark that Keener has shown (see Theorem 3.2 of [5]) that his results contained in [4] are applicable to equations which satisfy $(*)$. In [4], he investigates the nature of oscillatory solutions provided it is known that the equation is oscillatory.

\section{REF ERENCES}

1. G. B. Gustafson, The nonequivalence of oscillation and nondisconjugacy, Proc. Amer. Math. Soc. 25 (1970), 254-260. MR 44 \#1872.

2. - Eventual disconjugacy of self-adjoint fourth order linear differential equations, Proc. Amer. Math. Soc. 35 (1972), 187-192. MR 45 \# 7178.

3. M. Hanan, Oscillation criteria for third-order linear differential equations, Pacific J. Math. 11 (1961), 919-944. MR 26 \#2695.

4. M. S. Keener, Oscillatory solutions and multi-point boundary value functions for certain nth-order linear ordinary differential equations, Pacific J. Math. 51 (1974), 187-202.

5. - On the equivalence of oscillation and the existence of infinitely many conjugate points, Rocky Mountain J. Math. 5 (1975), 125-134.

6. W. Leighton and Z. Nehari, On the oscillation of solutions of selfadjoint linear differential equations of the fourth order, Trans. Amer. Math. Soc. 89 (1958), 325-377. MR 21 \#1429.

7. Z. Nehari, Disconjugate linear differential operators, Trans. Amer. Math. Soc. 129 (1967), 500-516. MR 36 \#2860.

8. J.R.Ridenhour, On the zeros of solutions of $n$th order linear differential equations, J. Differential Equations 16 (1974), 45-71.

9. T. L. Sherman, Properties of solutions of nth order linear differential equations, Pacific J. Math. 15 (1965), 1045-1060. MR 32 \#2654. 